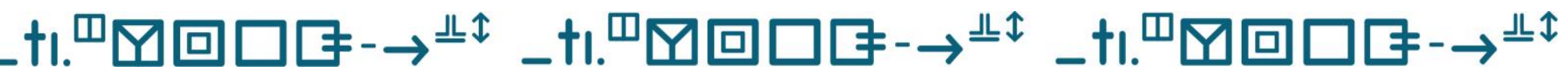

\title{
Formação de professores de Libras: ensino da escrita de sinais nas licenciaturas em Libras
}

\section{Education and training of Libras teachers: teaching sign language writing in undergraduate courses in Libras}

\footnotetext{
iD 9 Fabiane Ferreira da Silva Moraes

Universidade Federal de Goiás, Goiânia, Goiás, Brasil.
}

fabianemoraes@ufg.br

Resumo: O presente trabalho tem por objetivo responder ao seguinte questionamento: qual o espaço da escrita de sinais no currículo dos cursos de licenciatura em Libras ofertados por Instituições Federais de Ensino Superior? Para o desenvolvimento da pesquisa, optou-se pela pesquisa documental, de caráter qualitativo. A seleção dos cursos que formam o corpus foi realizada a partir de uma busca no site do e-Mec. Os resultados apontaram que o espaço da escrita de sinais no currículo dos cursos de licenciatura em Libras, ofertados por Instituições Federais de Ensino Superior, ainda é muito limitado e que, em alguns casos, o estudo da escrita de sinais nem ao menos faz parte da formação de professores de Libras. Nos cursos em que há a exigência do estudo da modalidade escrita da Libras, foram encontrados entraves como a carga horária reduzida e período em que as disciplinas são ofertadas.

Palavras-chave: Escrita de sinais. Formação. Currículo. Libras. 


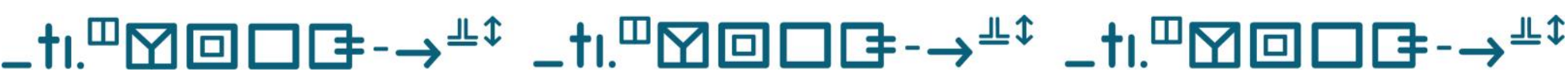

Formação de professores de Libras: o ensino da escrita de sinais nas licenciaturas em Libras

Fabiane Ferreira da Silva Moraes

Abstract: This paper aims to answer the following question: what is the space occupied by sign language writing in the syllabus of undergraduate teacher training courses in Libras offered by Federal Institutions of Higher Education? For the development of the research, documental research, qualitative in character, was chosen. The selection of the courses that make up the corpus of this research was carried out through a search on the e-Mec website. The results showed that the space occupied by sign language writing in the curriculum of undergraduate courses in Libras, offered by Federal Institutions of Higher Education, is still very limited and that, in some cases, the study of sign language writing is not even part of the education of Libras teachers. In the courses where the study the written form of Libras is required, obstacles were found such as reduced workloads and the period in which the subjects are offered.

Keywords: Sign language writing. Education. Syllabus. Libras.

Submetido em 05 de abril de 2021.

Aceito em 04 de junho de 2021.

Publicado em 04 de novembro de 2021. 


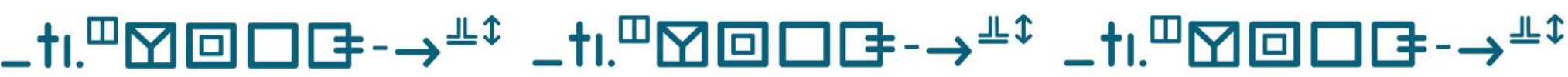

Formação de professores de Libras: o ensino da escrita de sinais nas licenciaturas em Libras

Fabiane Ferreira da Silva Moraes

\section{Introdução}

A Língua Brasileira de Sinais (Libras) foi reconhecida como meio legal de comunicação e expressão apenas em 2002, com a Lei $n^{\circ}$. 10.436. Contudo, o amparo legal para o uso da modalidade sinalizada (equivalente à fala nas línguas orais) não implicou no reconhecimento de que a Libras é dotada do mesmo valor e status que o português, a língua oficial do país. Um exemplo desta situação pode ser percebido na lei citada anteriormente, onde se lê que a língua de sinais "não poderá substituir a modalidade escrita da língua portuguesa" (BRASIL, 2002). Vale destacar que a língua de sinais não é ágrafa. Existem sistemas que possibilitam sua escrita e estudos que sustentam sua eficiência e relevância para os sujeitos surdos (BARROS, 2008; STUMPF, WANDERLEY, 2016; STUMPF, LUCHI, 2018; entre outros).

Um dos espaços de formação de professores de Libras são as licenciaturas em Libras. Esses cursos, além de sua importância acadêmica, são compreendidos na comunidade surda como uma conquista advinda das lutas e movimentos em prol do reconhecimento linguístico da Libras. Considerando-se a relevância dos referidos cursos, faz-se necessário compreender como eles estão estruturados e quais os componentes curriculares ofertados para os discentes. Tendo em vista a necessidade de delimitação da pesquisa, o presente trabalho tem por objetivo responder ao seguinte questionamento: qual o espaço da escrita de sinais 


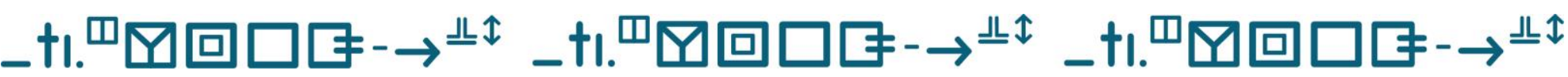

Formação de professores de Libras: o ensino da escrita de sinais nas licenciaturas em Libras

Fabiane Ferreira da Silva Moraes

(ES) no currículo dos cursos de licenciatura em Libras ofertados por Instituições Federais de Ensino Superior (IFES)?

Compreender o local da ES nesses cursos é importante já que os discentes formados poderão apoiar de modo significativo a difusão da escrita e de seus usos sociais ou perpetuar as concepções de desvalorização e subalternidade. O posicionamento dos futuros professores acerca da ES é relevante, pois levar a escrita de sinais para alunos surdos é uma possibilidade de valorização e incorporação de um elemento da cultura surda, de modo que as interações escritas no ambiente escolar ocorram também em língua de sinais e não exclusivamente em língua portuguesa (BARROS, 2008).

Além disso, há escassez de pesquisas sobre as instituições, cursos e vagas dedicadas às graduações em Libras e em Pedagogia Bilíngue no país. Isso porque, de acordo com Kumada e Prieto (2019, p. 81), os trabalhos acadêmicos estão voltados, em sua maioria, para os cursos “pioneiros da UFSC e do INES, invisibilizando as contribuições de outras IFES. Com isso, nota-se que, na prática e mesmo nas produções científicas, o contexto da formação de professores tem sido constantemente associado apenas a essas duas Ifes".

Na primeira parte do trabalho são apresentados alguns pressupostos teóricos sobre o currículo e formação de professores, fundamentados em Sacristán (2013). Também são discutidas questões sobre a formação de professores de 


\section{_t..}

Formação de professores de Libras: o ensino da escrita de sinais nas licenciaturas em Libras

Fabiane Ferreira da Silva Moraes

Libras (CERNY, QUADROS E BARBOSA, 2009; LODI e LACERDA, 2015) e acerca da escrita de sinais (BARROS, 2008; STUMPF e WANDERLEY, 2016, entre outros). Em seguida, apresentam-se a metodologia de pesquisa, a constituição do corpus e os critérios a serem avaliados no trabalho. A seção que segue é formada pela exposição dos dados analisados. Por fim, discorre-se sobre os resultados encontrados.

\section{Currículo, Libras e a Escrita de Sinais}

Conforme referenciam Lodi e Lacerda (2015, p. 287), a formação de professores de Libras começou a ser organizada no Brasil na década de 1990, período no qual as "metodologias específicas começaram a ser delineadas, considerando a materialidade visual, gestual e espacial desta língua". Como desdobramento da Lei $n^{\circ}$. 10.436/2002 e do Decreto $n^{\circ}$. $5.626 / 2005$, foram criados os primeiros cursos de licenciatura e bacharelado em Libras no Brasil, sendo que a primeira licenciatura em Letras: Libras foi ofertada em 2006, pela Universidade Federal de Santa Catarina, na modalidade à distância. Esse curso "tem sido a base para a proposição e implantação da maioria dos novos, fato que determina a hegemonia de um pensamento único sobre o perfil que se pretende formar como professor de Libras" (LODI, LACERDA, 2015, p. 291). Além disso, o Plano Viver sem Limite, lançado pelo governo federal em 2011, fomentou o surgimento desses cursos em todo o país, pois ele previa a criação de vinte e sete cursos de licenciatura em Libras, além de doze cursos de 


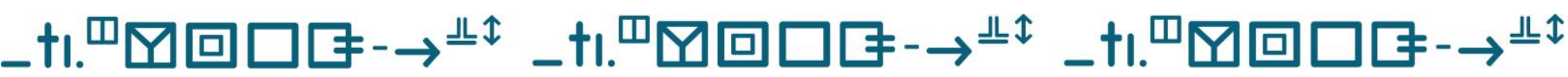

Formação de professores de Libras: o ensino da escrita de sinais nas licenciaturas em Libras

Fabiane Ferreira da Silva Moraes

Pedagogia na perspectiva bilíngue (BRASIL, 2014). Até 2014 haviam sido implementados vinte cursos superiores de Libras, que somados aos cinco já existentes antes do Plano Viver sem Limite, totalizavam vinte e cinco cursos (BRASIL, 2014).

Cabe observar que, historicamente, as discussões sobre a língua de sinais envolvem o cotejo entre valores distintos sobre a surdez - vista como marca cultural ou como deficiência. Atualmente, ainda que a legislação tenha reconhecido a Libras como língua, permanecem em questão as relações de poder entre a Libras e a língua portuguesa. Conforme exposto por Quadros (2012), mesmo nas propostas bilíngues, a língua portuguesa ainda é considerada como a língua superior e oficial, enquanto a língua de sinais é tida como um instrumento utilizado para que os discentes aprendam a língua dominante. Evidentemente, nos cursos de formação de professores de Libras a preocupação central é o ensino da língua de sinais, de modo que

nossas universidades têm privilegiado o modelo dos conteúdos culturais cognitivos na formação dos professores, e nesta mesma lógica os cursos de professores de Libras, a formação oferecida ao futuro professor dirige-se para o 'saber bem Libras' (domínio e fluência), 'saber sobre a Libras' (conhecimento sobre aspectos linguísticos), ter conhecimentos sobre desenvolvimento e aprendizagem de pessoas surdas e sobre os Estudos Surdos (LODI, LACERDA, 2015, p. 291). 


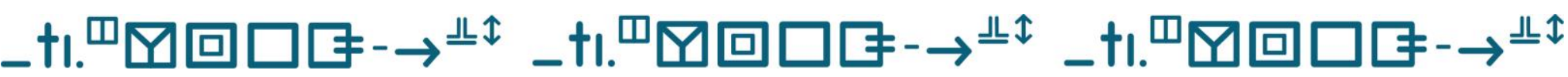

Formação de professores de Libras: o ensino da escrita de sinais nas licenciaturas em Libras

Fabiane Ferreira da Silva Moraes

Contudo, o "saber bem Libras", via de regra, é entendido como o domínio e fluência na modalidade sinalizada, não havendo a mesma preocupação com o domínio da modalidade escrita. As questões de poder e hegemonia da língua oficial do país podem ser notadas "nas escolas de surdos, que não acredita na escrita de sinais como forma de conhecimento legítimo. Diferentes argumentos são utilizados como 'ainda não está provado', ‘a sociedade usa o português!!!! para que mais uma língua???"' (SILVA, 2013, p. 55).

Sobre este aspecto, é interessante notar a concepção de Foucault de relações de poder, relações essas que estão enraizadas na sociedade e que perpassam os sujeitos (FOUCAULT, 1995). Se no passado as relações de poder impuseram a proibição do uso da língua de sinais, atualmente, por meio de sua elaboração e ajuste, elas permitem que o Estado conceda o direito de uso de uma modalidade da língua - a sinalizada - ao mesmo tempo em que o direito não se estende à escrita. Foucault (1995, p. 246) assinala que “[...] o exercício do poder não é um fato bruto, um dado institucional, nem uma estrutura que se mantém ou se quebra: ele se elabora, se transforma, se organiza, se dota de procedimento mais ou menos ajustados". Na perspectiva foucaultiana, o exercício do poder não se dá apenas pelo Estado, antes, o poder é exercido por todo o corpo social. Nesse sentido, podemos 


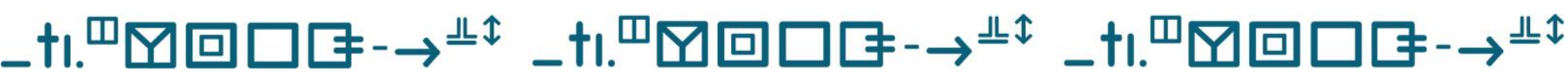

Formação de professores de Libras: o ensino da escrita de sinais nas licenciaturas em Libras

Fabiane Ferreira da Silva Moraes

notar as relações de poder presentes na construção dos currículos dos cursos de Letras: Libras.

Sacristán (2013) define o currículo como um percurso do que os alunos devem aprender e a ordem em que estes conteúdos serão apresentados. Assim, o currículo permite ordenar e delimitar os conteúdos a serem ensinados, além de possibilitar a coerência na aprendizagem e a regulação da prática didática. O autor destaca que, além dos conteúdos estudados, "quando começamos a desvelar suas origens suas implicações e os agentes envolvidos, os aspectos que o currículo condiciona e aqueles por ele condicionados, damonos conta de que nesse conceito se cruzam muitas dimensões" (SACRISTÁN, 2013, p. 16). Depreende-se então que a elaboração de um currículo é marcada por fatores culturais, políticos e econômicos (APPLE, 2002).

Nesse mesmo sentido, Sacristán (2013) explica que a construção de um currículo não é neutra. Ela envolve uma série de decisões que não são, evidentemente, as únicas possíveis. Nas palavras do autor:

A importância fundamental do currículo para a escolaridade reside no fato de que ele é a expressão do projeto cultural e educacional que as instituições de educação dizem que irão desenvolver com os alunos (e para eles) aquilo que consideram adequado. Por meio desse projeto institucional, são expressadas forças, interesses ou valores e preferências da sociedade, de determinados setores sociais, das famílias, dos grupos políticos etc (SÁCRISTÁN, 2013, p. 23). 


\section{_t..}

Formação de professores de Libras: o ensino da escrita de sinais nas licenciaturas em Libras

Fabiane Ferreira da Silva Moraes

Acerca das licenciaturas em Libras, Cerny, Quadros e Barbosa (2009, p. 8) apontam que "o currículo traduz marcas impressas de uma cultura nem sempre visíveis, mas que estão latentes nas relações sociais de uma época". Uma das formas de perceber estas marcas culturais pode ser a tentativa de responder o questionamento feito por Sacristán (2013, p. 23): "o que adotamos como conteúdo e o que deixamos de lado?". Observar, a partir da análise dos Projetos Pedagógicos dos Cursos (PPC), o que está sendo adotado como conteúdo e o que está sendo deixado de lado na formação de professores de Libras, pode trazer indícios destas marcas, mesmo em um espaço voltado para a valorização da língua de sinais.

Conforme referencia Silva (2013, p. 57), a partir dos Estudos Culturais, a ES é vista como um artefato cultural da comunidade surda, "influindo diretamente na sua subjetividade de forma afirmativa, construindo e constituindo as identidades culturais e sociais desta comunidade". A relação entre a ES e a cultura surda também pode ser vista em Perlin (2003, p. 135), pois a autora afirma que o uso da escrita "opera uma reterritorialização cultural, língua que será terra fértil para a expressão e transmissão do pensamento relacionando-a com a alteridade".

Sob ótica semelhante, Stumpf e Luchi (2018, p. 98-99) ressaltam que a ES possibilita "ao surdo reconhecer sua língua de sinais como real em status linguístico tal qual qualquer outra língua oral [...] poderão empoderar os surdos em suas pesquisas e em seu próprio reconhecimento linguístico". A importância da ES como possibilidade de ampliação dos usos da língua e como expressão da cultura surda também é sublinhada por Barros (2008, p. 20). De 


\section{_t..}

Formação de professores de Libras: o ensino da escrita de sinais nas licenciaturas em Libras

Fabiane Ferreira da Silva Moraes

acordo com a autora, a ES permite que os sujeitos surdos construam "seus próprios espaços sem precisar de permissão da cultura dominante".

No Brasil, são utilizados predominantemente dois sistemas de ES: o SignWriting (SW) e o Sistema Brasileiro de Escrita das Línguas de Sinais (ELiS). O SignWriting foi desenvolvido por Valérie Sutton, em 1974, nos Estados Unidos para notar os movimentos de dança, trata-se de "um sistema de escritura mista, majoritariamente fonográfico, do ponto de vista interno, mas possuindo uma leitura 'ideográfica' de um ponto de vista externo" (STUMPF, 2005, p. 52). Já o Sistema Brasileiro de Escrita das Línguas de Sinais foi criado por Mariângela Estelita Barros em sua dissertação de mestrado (1997) e aperfeiçoado em sua tese de doutorado (2008). O sistema é de ordem alfabética, ou seja, os símbolos são denominados visografemas "unidades mínimas (-ema) escritas (graf-) dos visemas (vis-), uma nomenclatura específica para a escrita dos elementos das LS, ou simplesmente como letras" (BARROS, 2008, p. 26).

\section{Metodologia}

Para o desenvolvimento da pesquisa, optou-se pela pesquisa documental. Prodanov (2013) explica que, neste tipo de pesquisa, são organizados materiais que se encontram dispersos e que esta seleção se constitui em uma nova fonte de pesquisa. Os materiais selecionados foram os documentos disponibilizados pelos cursos de Letras: Libras, a saber: Projeto Pedagógico dos Cursos, as grades curriculares e ementários. Para a escolha dos documentos, foram priorizados documentos originais, produzidos pelos 


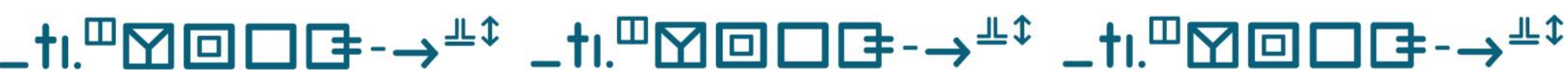

Formação de professores de Libras: o ensino da escrita de sinais nas licenciaturas em Libras

Fabiane Ferreira da Silva Moraes

cursos de Letras: Libras. A busca foi realizada apenas em arquivos públicos das IFES. A pesquisa de caráter qualitativo "considera que tudo pode ser quantificável, o que significa traduzir em números opiniões e informações para classificálas e analisá-las" (PRODANOV, 2013, p. 69).

A seleção dos cursos foi realizada a partir de uma busca no site do e-Mec ${ }^{1}$, tendo como critérios: cursos gratuitos de Libras, ofertados na modalidade presencial e à distância, com o grau de licenciatura. A busca teve como resultado uma listagem com 37 cursos. Desses, onze não foram analisados². A maior parte dos cursos era ofertada na modalidade presencial, apenas quatro eram à distância. Desse modo, formam o corpus de pesquisa os documentos dos cursos listados no quadro a seguir.

1 Busca realizada no dia 18/03/2020.

2 Após o levantamento inicial, fez-se necessário o estabelecimento de outros outros critérios: a) cursos ofertados por IFES; b) que tivessem em sua grade curricular a disciplina de ES; c) que tivessem disponibilizadas as informações do curso nos sites institucionais; d) que estivessem em funcionamento. Desse modo, não foram analisados o curso da Universidade Federal de Minas Gerais (UFMG) que ainda não havia sido iniciado; o curso da Universidade Federal de Sergipe (UFS), pois até o momento da pesquisa, as ementas, grade curricular ou PPC não estavam disponíveis no site da instituição; cursos oferecidos em universidades estaduais e municipal: Universidade do Estado do Pará (UEPA), três da Universidade Estadual do Piauí e um do Centro Universitário de Mineiros (Unifimes); o da Universidade Federal do Pará (UFPA), pois não constava a disciplina de ES no PPC do curso; o da Universidade comunitária da região de Chapecó (UNOCHAPECÓ), já que o curso está em processo de desativação; o da Universidade Federal do Mato Grosso (UFMT), por problemas no site institucional e o curso do Instituto Federal de Educação, Ciência e Tecnologia de Santa Catarina (IFSC), por ser de Pedagogia Bilíngue Libras/Português. 


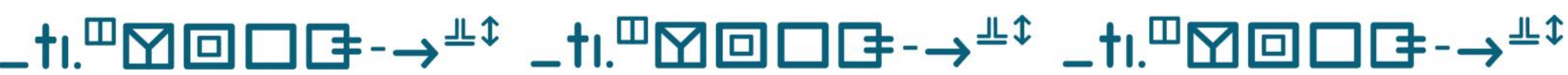

Formação de professores de Libras: o ensino da escrita de sinais nas licenciaturas em Libras

Fabiane Ferreira da Silva Moraes

\section{Quadro 1 - Relação de instituições que ofertam os cursos analisados na modalidade presencial.}

\begin{tabular}{|c|c|}
\hline 1. Universidade Federal do Acre (UFAC) & 12. Universidade Federal do Paraná (UFPR) \\
\hline 2. Universidade Federal de Alagoas (UFAL) & $\begin{array}{l}\text { 13. Universidade Federal de Pernambuco } \\
\text { (UFPE) }\end{array}$ \\
\hline 3. Universidade Federal da Amazônia (UFAM) & 14. Universidade Federal do Piauí (UFPI) \\
\hline $\begin{array}{l}\text { 4. Universidade Federal Rural da Amazônia } \\
\text { (UFRA) }\end{array}$ & $\begin{array}{l}\text { 15. Universidade Federal do Recôncavo da } \\
\text { Bahia (UFRB) }\end{array}$ \\
\hline 5. Universidade Federal do Amapá (UNIFAP) & $\begin{array}{l}\text { 16. Universidade Federal do Rio de Janeiro } \\
\text { (UFRJ) }\end{array}$ \\
\hline 6. Universidade Federal do Cariri (UFCA) & $\begin{array}{l}\text { 17. Universidade Federal do Rio Grande do } \\
\text { Norte (UFRN) }\end{array}$ \\
\hline 7. Universidade Federal do Ceará (UFC) & 18. Universidade Federal de Rondônia (UNIR) \\
\hline $\begin{array}{l}\text { 8. Universidade Federal de Campina Grande } \\
\text { (UFCG) }\end{array}$ & $\begin{array}{l}\text { 19. Universidade Federal Rural do Semi-Árido } \\
\text { (UFERSA) }\end{array}$ \\
\hline 9. Universidade Federal de Goiás (UFG) & $\begin{array}{l}\text { 20. Universidade Federal de Santa Catarina } \\
\text { (UFSC) }\end{array}$ \\
\hline 10. Universidade Federal de Juiz de Fora (UFJF) & 21. Universidade Federal do Tocantins (UFT) \\
\hline 11. Universidade Federal do Maranhão (UFMA) & $\begin{array}{l}\text { 22. Universidade Federal de Uberlândia } \\
\text { (UFU) }\end{array}$ \\
\hline
\end{tabular}

Fonte: Elaborado pela autora com base em e-Mec (2021).

Descrição da imagem: Quadro intitulado "Relação de instituições que ofertam os cursos analisados na modalidade presencial". O quadro é composto pelo nome de 22 universidades federais que oferecem licenciaturas em Libras, na modalidade presencial. 


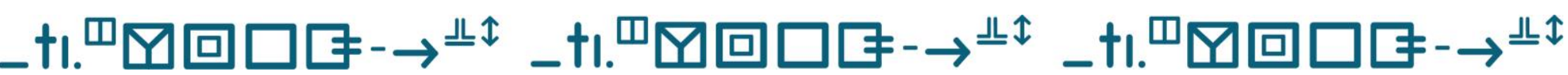

Formação de professores de Libras: o ensino da escrita de sinais nas licenciaturas em Libras

Fabiane Ferreira da Silva Moraes

Quadro 2 - Relação de instituições que ofertam os cursos analisados na modalidade à distância.

\begin{tabular}{|l|l|}
\hline $\begin{array}{l}\text { 1. Universidade Federal da Grande Dourados } \\
\text { (UFGD) }\end{array}$ & 3. Universidade Federal da Paraíba (UFPB) \\
\hline $\begin{array}{l}\text { 2. Instituto Federal de Educação, Ciência e e } \\
\text { Tecnologia do Norte de Minas Gerais (IFNMG) }\end{array}$ & $\begin{array}{l}\text { 4. Universidade Federal de Santa Catarina } \\
\text { (UFSC) }\end{array}$ \\
\hline
\end{tabular}

Fonte: Elaborado pela autora com base em e-Mec (2021).

Descrição da imagem: Quadro intitulado "Relação de instituições que ofertam os cursos analisados na modalidade presencial". O quadro é composto pelo nome de quatro universidades federais que oferecem licenciaturas em Libras, na modalidade à distância.

Em seguida, foram realizadas buscas pelos cursos de licenciatura em Libras nos sites institucionais de cada IFES listada. A pesquisa nos sites consistiu na verificação do Projeto Pedagógico do Curso, da grade curricular e do ementário. Em seguida, foram selecionados os dados para a análise, a saber: nome do curso, nome das disciplinas de ES, quantidade de disciplinas por curso, carga horária das disciplinas de ES, fluxo da disciplina, se a disciplina é obrigatória ou não, ementa e referencial bibliográfico. Os dados foram organizados em uma tabela que, devido à sua extensão, não foi anexada no presente artigo. Esses dados estão representados, de forma resumida, nos gráficos de 1 a 5. 


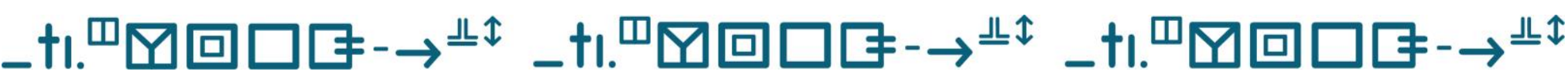

Formação de professores de Libras: o ensino da escrita de sinais nas licenciaturas em Libras

Fabiane Ferreira da Silva Moraes

\section{Análise dos dados}

O primeiro aspecto observado refere-se à nomenclatura das licenciaturas. Segundo os dados listados no site do e-Mec, dos vinte e dois cursos presenciais, apenas quatro não são denominados Letras: Libras (ver gráfico 1). O primeiro é o da UFRB, chamado Letras - Libras/Língua Estrangeira. O segundo é o da UNIFAP denominado Letras - Libras Português. O terceiro é o da UFU, nominado Letras - Língua Portuguesa com domínio de Libras e por fim o da UFRN intitulado Letras - Língua Portuguesa e Libras. Dos quatro cursos à distância, dois são denominados Letras: Libras (IFNMG, UFSC); já na UFPB e UFGD os cursos são nomeados Letras - Língua Portuguesa e Libras (ver gráfico 2).

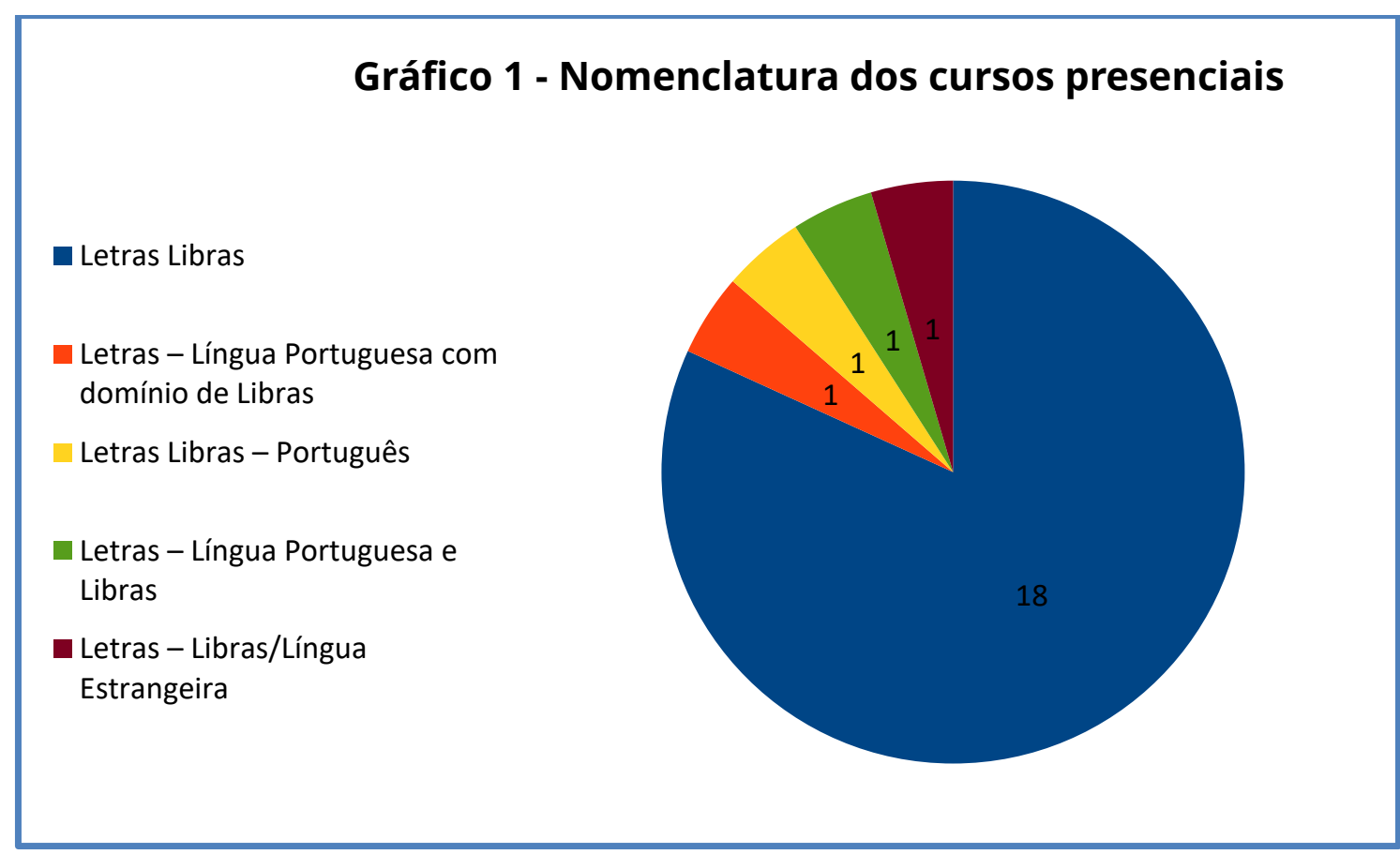

Fonte: Elaborado pela autora (2021).

Descrição da imagem: gráfico circular, no formato de pizza, com os nomes dos cursos presenciais e a quantidade de cursos com cada nome. Letras: Libras: 18 cursos na cor azul; Letras - Língua 


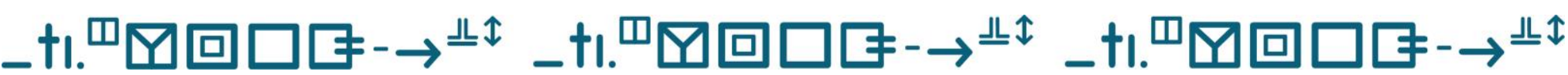

Formação de professores de Libras: o ensino da escrita de sinais nas licenciaturas em Libras

Fabiane Ferreira da Silva Moraes

Portuguesa com domínio de Libras na cor laranja: 1 curso; Letras Libras - Português na cor amarela: 1 curso; Letras - Língua Portuguesa e Libras: 1 curso na cor verde; e Letras Libras/Língua Estrangeira: 1 curso na cor vermelha.

\section{Gráfico 2 - Nomenclatura dos cursos à distância}

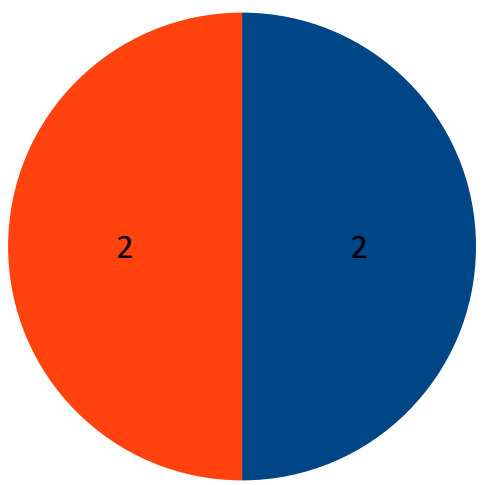

- Letras Libras

- Letras - Língua Portuguesa e Libras

Fonte: Elaborado pela autora (2021).

Descrição da imagem: gráfico circular, no formato de pizza, com os nomes dos cursos à distância e a quantidade de cursos com cada nome. Letras: Libras: 2 cursos na cor azul; Letras - Língua Portuguesa e Libras: 1 curso na cor laranja.

Em seguida, a análise se deteve na quantidade de disciplinas de ES obrigatórias ofertadas por cada IFES, assim como expresso no gráfico 3. Os cursos presenciais contam, predominantemente, com duas disciplinas de escrita de sinais, número que foi verificado em quatorze instituições, a saber: UFC, UFPR, UFERSA, UFSC, UFAL, UFCA, UFPI, UFMA, UFAC, UFPE, UFT, UFCG, UNIR, UNIFAP. Em três instituições é ofertada apenas uma disciplina obrigatória de ES, são elas: UFRJ, UFAM, UFU. Dois dos cursos analisados ofertam três disciplinas obrigatórias de ES: UFRA e UFG. Em três instituições não existem disciplinas obrigatórias de ES, 


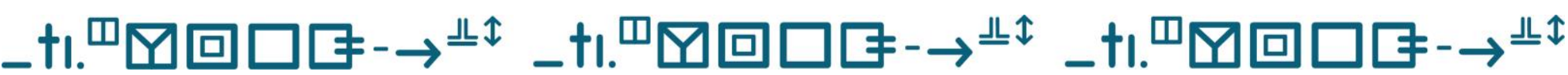

Formação de professores de Libras: o ensino da escrita de sinais nas licenciaturas em Libras

Fabiane Ferreira da Silva Moraes

apenas optativas: UFRN (3 optativas), UFRB (1 optativa), UFJF (1 oficina). Entre os cursos à distância, o ensino de ES é obrigatório e predomina a oferta de 3 disciplinas, apenas no curso do IFNMG são ministradas duas disciplinas.

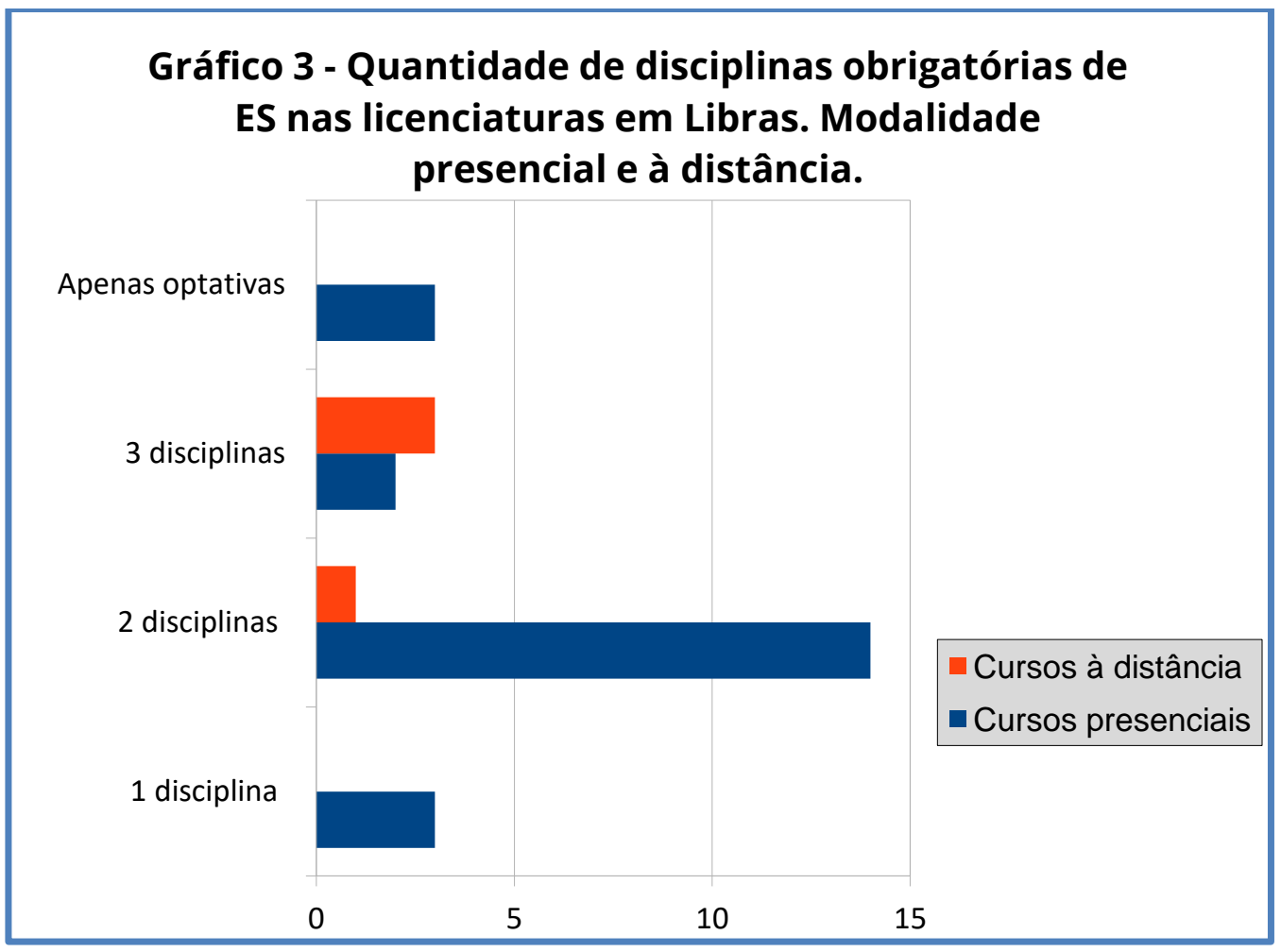

Fonte: Elaborado pela autora (2021).

Descrição da imagem: gráfico em barras horizontais, com a quantidade de disciplinas obrigatórias de ES nas licenciaturas em Libras. Em 3 cursos presenciais: apenas disciplinas optativas; em 3 cursos à distância e 2 presenciais: 3 disciplinas obrigatórias; em 1 curso à distância e 14 presenciais: 2 disciplinas obrigatórias; em 3 cursos presenciais: 1 disciplina obrigatória.

Evidentemente, o número de disciplinas por si só não é um indicativo do papel da ES nos cursos, isso porque pode haver uma diferença significativa na carga horária de cada componente curricular. Assim, o próximo aspecto observado foi o quantitativo de horas obrigatórias em cada curso, ilustrado no gráfico 4. Considerando que as IFES ofertam 


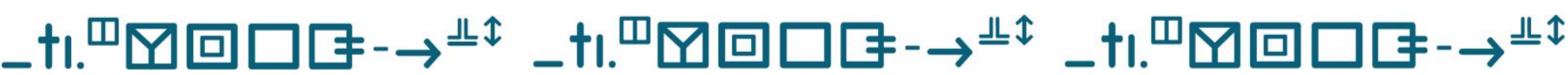

Formação de professores de Libras: o ensino da escrita de sinais nas licenciaturas em Libras

Fabiane Ferreira da Silva Moraes

números diferentes de disciplinas, optou-se por somar a carga horária em cada curso.

Nas licenciaturas presenciais, o curso com menor carga horária foi o da UFRJ, com 30 horas e o com maior carga horária é o da UFG, com 192 horas. Nos cursos à distância, nota-se que todos possuem mais de 100 horas de ensino de ES. A instituição com menor carga horária é o IFNMG, com 160 horas e a maior carga horária é a UFSC, com 216 horas.

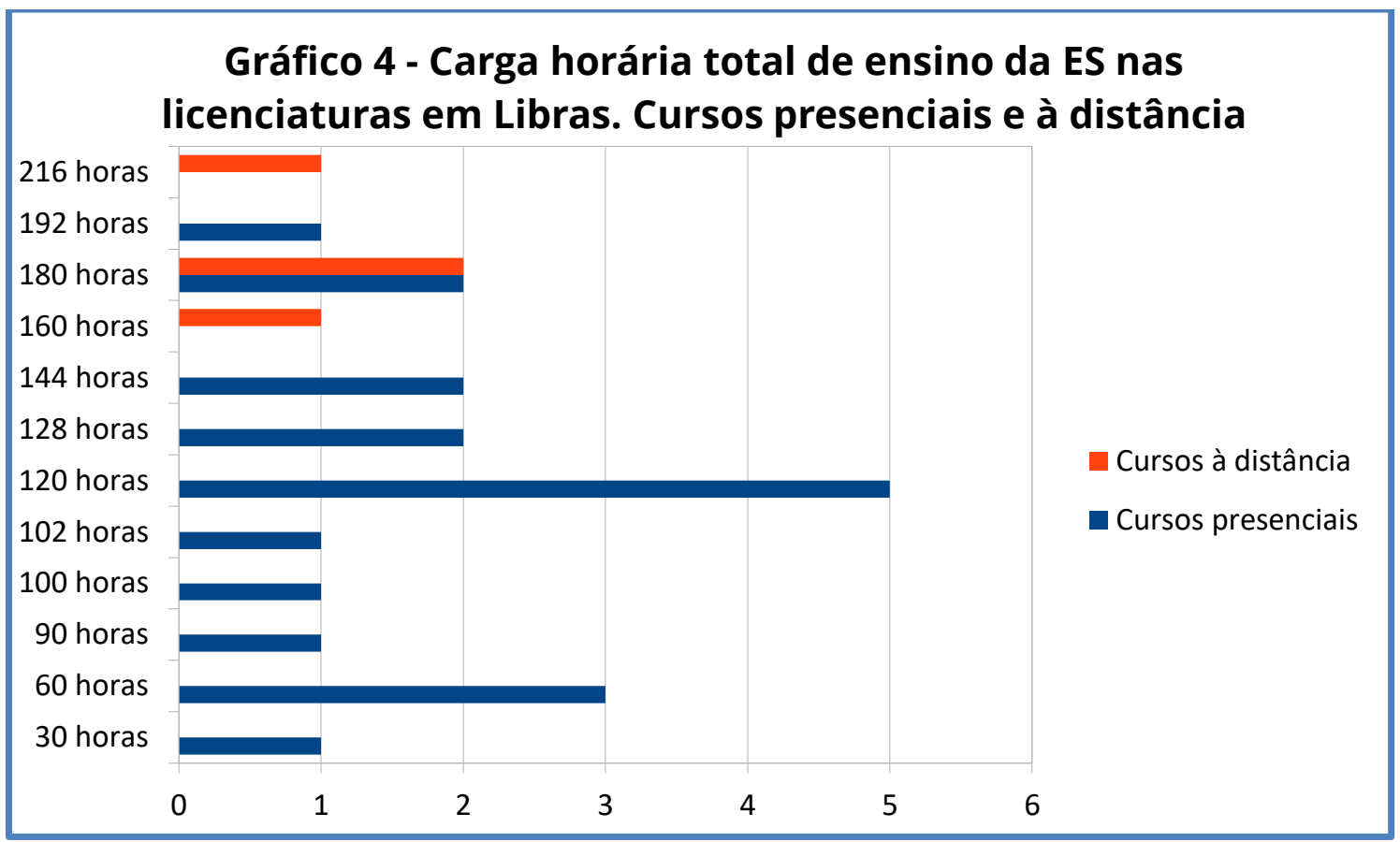

Fonte: Elaborado pela autora (2021).

Descrição da imagem: gráfico em barras horizontais com a carga horária total de ensino da ES nas licenciaturas em Libras. 216 horas: 1 curso à distância; 192 horas: 1 curso presencial; 180 horas: 2 cursos presenciais e 2 à distância; 160 horas: 1 curso à distância; 144 horas: 2 cursos presencias; 128 horas: 2 cursos presencias; 120 horas: 5 cursos presencias; 102 horas, 100 horas e 90 horas e 30 horas: 1 curso presencial em cada; 60 horas: 3 cursos presencias.

Além das disciplinas obrigatórias, a UFC, UFERSA, UFCA e a UFRJ possibilitam que os alunos façam uma disciplina 


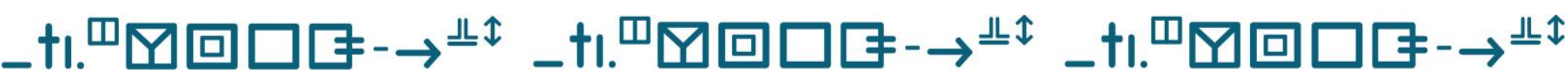

Formação de professores de Libras: o ensino da escrita de sinais nas licenciaturas em Libras

Fabiane Ferreira da Silva Moraes

optativa. Contudo, um aspecto chamou a atenção: na UFERSA, as disciplinas são ofertadas no sétimo e no oitavo período, e há uma optativa de ES 3. Mas, se o aluno cursa ES 2 no oitavo período como fará uma optativa? Na UFRN, as 3 disciplinas são optativas, ou seja, é facultativo ao aluno aprender ou não ES durante a licenciatura. Na UFRB, também é opcional o estudo da ES, e o curso oferta apenas uma disciplina, com 51 horas. Nos cursos à distância, todas as disciplinas são obrigatórias. Acerca do fluxo de disciplinas, chamou a atenção o fato de que, em uma instituição, a disciplina de ES é disponibilizada no oitavo período e em duas IFES o estudo da ES só é iniciado no sétimo período.

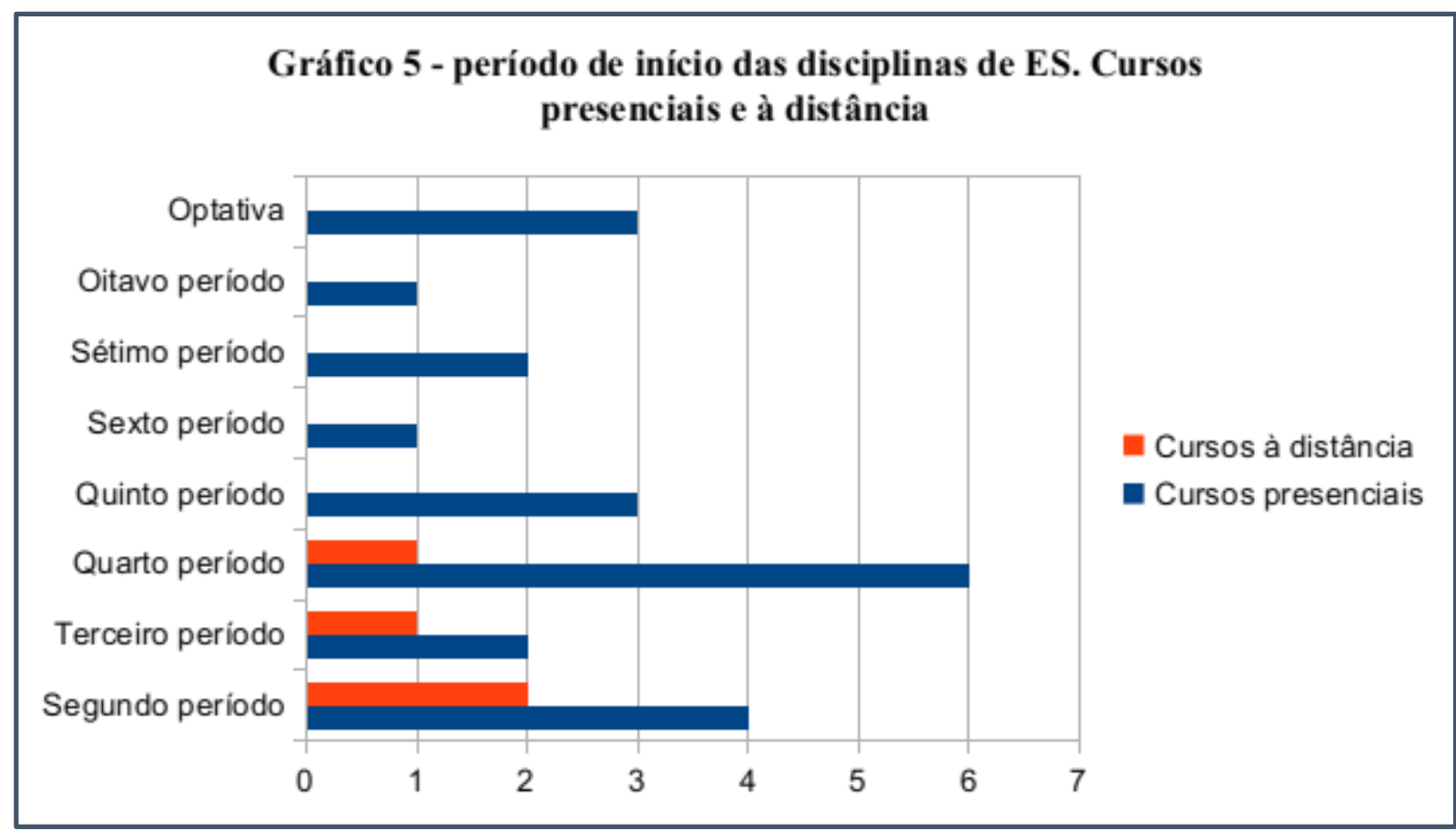

Fonte: Elaborado pela autora (2021).

Descrição da imagem: gráfico em barras horizontais com o período de início das disciplinas de ES, em cursos presenciais e à distância. Apenas optativas: 3 cursos presenciais; oitavo período: 1 curso presencial; sétimo período: 2 cursos presenciais; sexto período: 1 curso presencial; quinto 


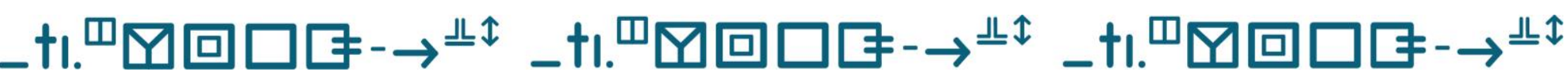

Formação de professores de Libras: o ensino da escrita de sinais nas licenciaturas em Libras

Fabiane Ferreira da Silva Moraes

período: 3 cursos presenciais; quarto período: 1 curso à distância e 6 presenciais; terceiro período: 1 curso à distância e 2 presenciais; segundo período: 2 cursos à distância e 4 presenciais.

Acerca do sistema de escrita ensinado nos cursos presenciais, observou-se a predominância do SW. Os primeiros documentos analisados foram as ementas da disciplina, a partir delas, notou-se que dez cursos presenciais evidenciam o ensino de SW, outros sistemas não são listados em nenhuma ementa. Oito cursos não citam o nome dos sistemas ensinados nas ementas, nesses casos, foram observadas a bibliografia básica das disciplinas. Em três cursos, mesmo após a análise da bibliografia, não foi possível identificar qual o sistema ensinado. As ementas e bibliografias básicas de quatro cursos não estavam disponíveis nos sites institucionais.

Quanto aos cursos à distância, apenas a ementa de um curso indicava o ensino dos dois sistemas, contudo a análise da bibliografia trazia obras de apenas um sistema - o SW. Outro curso também não trazia indicação na ementa, mas a bibliografia apontou para o SW, os demais cursos não traziam bibliografia específica. Os limites da pesquisa não permitiram verificar se o que é realmente ensinado condiz ou não com tais documentos.

Por fim, chamou a atenção o fato de que algumas IFES traziam ementas iguais, o que foi identificado em três casos. No primeiro, foram identificadas ementas iguais para as disciplinas de ES 1, 2 e 3 em três universidades. No segundo exemplo, notou-se que duas IFES também utilizam ementas 


\section{_t..}

Formação de professores de Libras: o ensino da escrita de sinais nas licenciaturas em Libras

Fabiane Ferreira da Silva Moraes

modalidade escrita da língua que ensinarão. Além disso, o fluxo das disciplinas, na constituição do currículo, também é relevante e traz impactos para a formação dos discentes. Os inúmeros usos da ES como seu emprego para elaboração de trabalhos, para aprendizagem da modalidade sinalizada, para a comunicação escrita sem a necessidade de tradução para o português, entre outros, poderiam ser de grande valia para os acadêmicos, caso o sistema de escrita fosse ensinado nos períodos iniciais do curso. Ademais, quando a oferta tem início no sétimo ou oitavo período, as possibilidades dos discentes desenvolverem atividades no estágio que envolvam também a modalidade escrita da língua e não apenas a sinalizada são muito restritas. As desvantagens também se estendem para a limitação da elaboração de trabalhos finais de curso que envolvam pesquisas sobre a escrita de sinais ou mesmo que sejam escritos em ES e não em português.

Retomando a afirmação de Sacristán (2013) de que o currículo não é neutro e de que é preciso indagar sobre o que é elencado como conteúdo e o que é deixado de lado, cabe refletir sobre quais os valores, quais as concepções sobre a escrita e sobre a língua de sinais permeiam a construção dos currículos de formação de professores de Libras, bem como sobre os desdobramentos do espaço da ES nos currículos das licenciaturas para as práticas docentes dos graduandos. Os apontamentos levantados na presente pesquisa podem servir como ponto de partida para uma melhor compreensão dessas questões. 


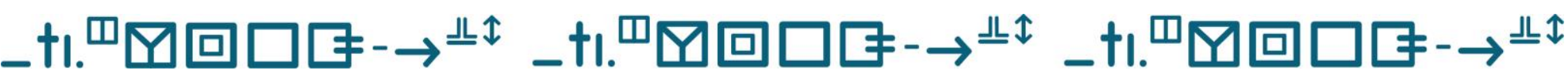

Formação de professores de Libras: o ensino da escrita de sinais nas licenciaturas em Libras

Fabiane Ferreira da Silva Moraes

\section{Referências}

APPLE, M. W. A POLITICA DO CONHECIMENTO OFICIAL: FAZ SENTIDO A IDEIA DE UM CURRÍCULO NACIONAL? IN: MOREIRA, A. F. B; SILVA, T. T. (ORG.). CURRÍCULO, CULTURA E SOCIEDADE. SÃo PAULO: CORTEZ, 2002.

BARROS, M. E. ELIS - ESCRITA DAS LÍNGUAS DE SINAIS: PROPOSTA TEÓRICA E VERIFICAÇÃO PRÁTICA. 2008. 192F. TESE (DOUTORADO EM LINGUÍSTICA) - CENTRO DE COMUNICAÇÃO E EXPRESSÃO, UNIVERSIDADE FEDERAL DE SANTA CATARINA, FLORIANÓPOLIS.

\section{BRASIL. CADASTRO NACIONAL DE CURSOS E INSTITUIÇÕES DE} EDUCAÇÃO SUPERIOR - CADASTRO E-MEC. DISPONÍVEL EM: <HTTPS://EMEC.MEC.GOV.BR/>. ACESSO EM: 04 DE ABRIL DE 2020. BRASIL. LEI No 10.436, DE 24 DE ABRIL DE 2002. DISPÕE SOBRE A LÍNGUA BRASILEIRA DE SINAIS - LIBRAS E DÁ OUTRAS PROVIDÊNCIAS. IN: DIÁRIO OFICIAL dA UNIÃO, N. 79, SEÇÃO 1, DE 25/4/2002. P. 23. DISPONÍVEL EM: <HTTP://PESQUISA.IN.GOV.BR/IMPRENSA/JSP/VISUALIZA/INDEX.JSP?JORNA L=1\&PAGINA=23\&DATA=25/04/2002>. ACESSO EM: 10 JUN. 2020. BRASIL. SECRETARIA DE DIREITOS HUMANOS. VIVER SEM LIMITES PLANO NACIONAL DOS DIREITOS DA PESSOA COM DEFICIÊNCIA. BRASÍLIA, 2014. DISPONÍVEL EM: <HTTPS://WWW.MPES.MP.BR/ARQUIVOS/ANEXOS/53630C9C-3EDA46D3-B013-FB83152E3495.PDF>. ACESSO EM 15 DE SET. DE 2020.

CERNY, R. Z.; QUADROS, R. M.; BARBOSA, H. FORMAÇÃO DE PROFESSORES DE LETRAS-LIBRAS: CONSTRUINDO O CURRÍCULO. ECURRICULUM. SÃo PAULO, v. 4, N. 2, JUN 2009. DISPONÍVEL EM: 


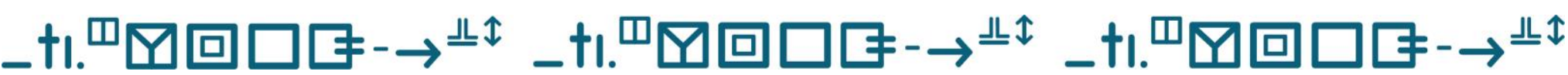

Formação de professores de Libras: o ensino da escrita de sinais nas licenciaturas em Libras

Fabiane Ferreira da Silva Moraes

<HTTP://KEN.PUCSP.BR/CURRICULUM/ARTICLE/VIEW/3230/2148>. ACESSO EM: 15 DE SET. DE 2020.

FOUCAULT, M. O SUJEITO E O PODER. IN: DREYFUS, HUBERT L.; RABINOW, PAUL. (ORG.) MICHAEL FOUCAULT: UMA TRAJETÓRIA FILOSÓFICA (PARA ALÉM DO ESTRUTURALISMO E DA HERMENÊUTICA). RIO DE JANEIRO: FORENSE UNIVERSITÁRIA, 1995.

INSTITUTO FEDERAL DO NORTE DE MINAS GERAIS. PROJETO PEDAGÓGICO DO CURSO DE LETRAS - LÍNGUA BRASILEIRA DE SINAIS. DISPONÍVEL EM: <HTTP://DOCUMENTO.IFNMG.EDU.BR/ACTION.PHP?KT_PATH_INFO=KTCO RE.ACTIONS.DOCUMENT.VIEW\&FDOCUMENTID=20757>. ACESSO EM: 03 DE OUT. DE 2020.

KUMADA, K. M. O.; PRIETO, R. G. DESDOBRAMENTOS DA POLÍTICA DE EDUCAÇÃO SUPERIOR PARA FORMAÇÃO DO DOCENTE DE LIBRAS. CAdernos de Pesquisa, SÃo PAUlo, V. 49, N. 173, P. 64-84, SET. 2019. DISPONÍVEL EM: <HTTP://WWW.SCIELO.BR/SCIELO.PHP?SCRIPT=SCI_ARTTEXT\&PID=S010 0-15742019000300064\&LNG=PT\&NRM=ISO>. ACESSO EM: 15 DE SET. DE 2020.

LODI, A. C. B.; LACERDA, C. B. F. FORMAÇÃO DE PROFESSORES DE LÍNGUA BRASILEIRA DE SINAIS: REFLEXÕES SOBRE O IMPACTO DESTA AÇÃO PARA A EDUCAÇÃO. EduCAÇÃo E FILOSOFIA. UBERLÂNDIA, V. 29, N. ESPECIAL, P. 279 - 299, 2015. DISPONÍVEL EM: <HTTP://WWW.SEER.UFU.BR/INDEX.PHP/EDUCACAOFILOSOFIA/ARTICLE/V IEW/29983/18249>. ACESSO EM: 15 DE SET. DE 2020.

PERLIN, G. T. T. O SER E O ESTAR SENDO SURDOS: ALTERIDADE, DIFERENÇA E IDENTIDADE. 2003. 156F. TESE (DOUTORADO EM 


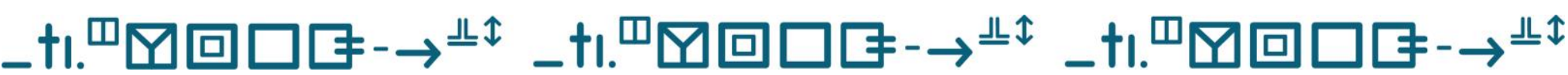

Formação de professores de Libras: o ensino da escrita de sinais nas licenciaturas em Libras

Fabiane Ferreira da Silva Moraes

EDUCAÇÃO) - FACULDADE DE EDUCAÇÃO, UNIVERSIDADE FEDERAL DO RIO GRANDE DO SUL, PORTO ALEGRE.

PRODANOV, C. C. MEtOdologia do tRABalHo Científico: MÉTODOS E TÉCNICAS DA PESQUISA E DO TRABALHO ACADÊMICO. NOVO HAMBURGO: FEEVALE, 2013.

QUADROS, R. M. O “BI” EM BILINGUISMO NA EDUCAÇÃO DE SURDOS. IN: FERNANDES, EULÁLIA. SURDEZ E BILINGUISMO. PORTO ALEGRE, MEDIAÇÃO, 2012.

SACRISTÁN, J. G. O QUE SIGNIFICA O CURRÍCULO? IN: SACRISTÁN, J. G. (ORG.). SABERES E INCERTEZAS SOBRE O CURRÍ́cULO. PORTO ALEGRE: PENSO, 2013.

SILVA, E. V. L. S. NARRATIVAS DE PROFESSORES DE SURDOS SOBRE A ESCRITA DE SINAIS. 2013. 113F. DISSERTAÇÃo (MESTRADO EM EDUCAÇÃO) - FACULDADE DE EDUCAÇÃO, UNIVERSIDADE FEDERAL DO RIO GRANDE DO SUL, PORTO ALEGRE.

STUMPF, M. R. APRENDIZAGEM DE ESCRITA DE LíNGUA DE SINAIS PELO SISTEMA SIGNWRITING: LÍNGUAS DE SINAIS NO PAPEL E NO COMPUTADOR. 2005. 330F. TESE (DOUTORADO EM INFORMÁTICA NA EDUCAÇÃO) - FACULDADE DE EDUCAÇÃO, UNIVERSIDADE FEDERAL DO RIO GRANDE DO SUL, PORTO ALEGRE.

STUMPF, M. R; WANDERLEY, D. C. QUEM FALA PORTUGUÊS, ESCREVE EM PORTUGUÊS. QUEM FALA INGLÊS, ESCREVE EM INGLÊS. OS SURDOS: ESCREVEM EM QUE LÍNGUA?. IN: REVISTA LETRAS RARAS, CAMPINA GRANDE, V. 5, N. 1, P. 93-107, 2016. HTTP://REVISTAS.UFCG.EDU.BR/CH/INDEX.PHP/RLR/ARTICLE/VIEW/586. ACESSO EM: 09 DE JUNHO DE 2019. 


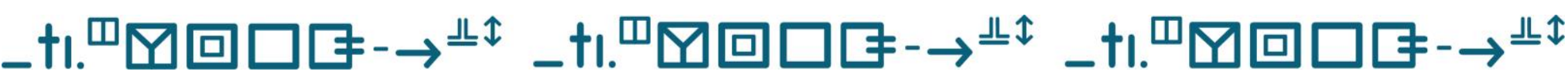

Formação de professores de Libras: o ensino da escrita de sinais nas licenciaturas em Libras

Fabiane Ferreira da Silva Moraes

STUMPF, M. R; LUCHI, M. ASPECTOS LINGUÍSTICOS DA ESCRITA DE SINAIS. IN: STUMPF, M. R; QUADROS, R. M. (ORGS.). ESTUDOS DA LÍNGUA BRASILEIRA DE SINAIS IV. FLORIANÓPOLIS: INSULAR, 2018. UNIVERSIDADE FEDERAL DO ACRE. EMENTÁRIO. DISPONIVEL EM: <HTTPS://PORTAL.UFAC.BR/EMENTARIO/CURRICULO.ACTION;JSESSIONID= 6601E9E25D02E1ABFEA5AFC741798188?V=303>. ACESSO EM: 03 DE OUT. DE 2020.

UNIVERSIDADE FEDERAL DE ALAGOAS. PPC DO CURSO DE LETRAS-LIBRAS. DISPONÍVEL EM:

<HTTP://WWW.UFAL.EDU.BR/UNIDADEACADEMICA/FALE/GRADUACAO/LL UFAL/PROJETO-PEDAGOGICO/PPC-DO-CURSO-DE-LETRAS-LIBRAS/VIEW>. ACESSO EM: 03 DE OUT. DE 2020.

UNIVERSIDADE FEDERAL DO AMAPÁ. PPC E DISCIPLINAS OFERTADAS. DISPONÍVEL EM: <HTTPS://WWW2.UNIFAP.BR/LETRASLIBRAS/DISCIPLINAS-OFERTADAS/>. ACESSO EM: 03 DE OUT. DE 2020.

UNIVERSIDADE FEDERAL DO AMAZONAS. PROJETO POLítICO PEDAGÓGICO DO CURSO DE LETRAS - LIBRAS. DISPONÍVEL EM: <HTTPS://BIBLIOTECA.UFAM.EDU.BR/ATTACHMENTS/ARTICLE/256/PPP\% 20LETRAS\%20LIBRAS\%20UFAM\%20\%2025.07.2014NEW.PDF>. ACESSO EM: 03 DE OUT. DE 2020.

UNIVERSIDADE FEDERAL DE CAMPINA GRANDE. RESOLUÇÃo 08/2016, DE 16 DE AGOSTO DE 2016. DISPONÍVEL EM: <HTTP://WWW.UFCG.EDU.BR/ COSTA/RESOLUCOES/RES_16082016.PD F>. ACESSO EM: 03 DE OUT. DE 2020. 


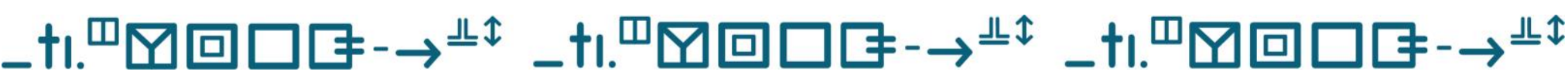

Formação de professores de Libras: o ensino da escrita de sinais nas licenciaturas em Libras

Fabiane Ferreira da Silva Moraes

UNIVERSIDADE FEDERAL DO CARIRI. LETRAS-LIBRAS DISPONIVEL EM: < HTTPS://WWW.UFCA.EDU.BR/CURSOS/GRADUACAO/LETRASLIBRAS/>. ACESSO EM: 03 DE OUT. DE 2020.

UNIVERSIDADE FEDERAL DO CEARÁ. PRojeto PedAGógICo do CURSO. DISPONÍVEL EM:

<HTTPS://SI3.UFC.BR/SIGAA/PUBLIC/CURSO/PPP.JSF?LC=PT_BR\&ID=137 1493>. ACESSO EM: 03 DE OUT. DE 2020.

UNIVERSIDADE FEDERAL DA GRANDE DOURADOS. PROJETO PEDAGÓGICO DO CURSO DE LETRAS - LIBRAS, LICENCIATURA.

DISPONÍVEL EM:

<HTTP://FILES.UFGD.EDU.BR/ARQUIVOS/ARQUIVOS/78/COGRAD/PPC \%20LIBRAS\%20LICENCIATURA_.PDF>. ACESSO EM: 03 DE OUT. DE 2020.

UNIVERSIDADE FEDERAL DE JUÍZ DE FORA. PRoJeTo PEDAGOGICO DO CURSO DE GRADUAÇÃO LETRAS-LIBRAS (LICENCIATURA). DISPONÍVEL EM:

< HTTPS://WWW.UFJF.BR/LETRASLIBRAS/FILES/2016/04/PPC-LETRASLIBRAS-APROVADO-23-08-2018-1.PDF>. ACESSO EM: 03 DE OUT. DE 2020.

UNIVERSIDADE FEDERAL DO MARANHÃO. PROJETO POLÍTICO PEDAGÓGICO CURSO DE GRADUAÇÃo EM LETRAS - LICENCIATURA EM LÍNGUA BRASILEIRA DE SINAIS. DISPONÍVEL EM: <HTTP://WWW.UFMA.BR/PORTALUFMA/ARQUIVO/THIBLVQMLKFDNFU .PDF>. ACESSO EM: 03 DE OUT. DE 2020.

UNIVERSIDADE FEDERAL DO PARÁ. RESOLUÇÃO 4.661, DE 28 DE ABRIL DE 2015. DISPONÍVEL EM:

$<W W W . P P C . P R O E G$.UFPA.BR/VIEW/_PAGINAS/_DOWNLOADANEXOPROJET O.PHP?ID=237\&IDPROJETO=30>. ACESSO EM: 03 DE OUT. DE 2020. 


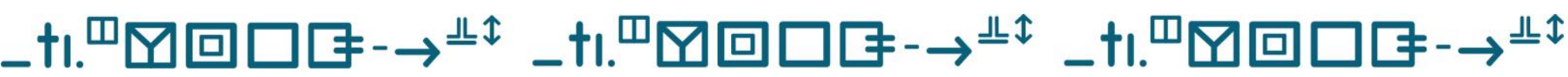

Formação de professores de Libras: o ensino da escrita de sinais nas licenciaturas em Libras

Fabiane Ferreira da Silva Moraes

UNIVERSIDADE FEDERAL DO PARANÁ. CURRÍcULO dO CURSO.

DISPONIVEL EM: <HTTP://WWW.LETRASLIBRAS.UFPR.BR/CURRICULO-

2018-RESOLUCAO-1418-CEPE/>. ACESSO EM: 03 DE OUT. DE 2020.

UNIVERSIDADE FEDERAL DE PERNAMBUCO. CURRÍ́cULO dO

CURSO DE GRADUAÇÃO/ LICENCIATURA EM LETRAS - LIBRAS.

DISPONÍVEL EM:

< HTTPS://WWW.UFPE.BR/DOCUMENTS/38970/411209/ESTRUTURA-

CURRICULAR-DO-CURSO-DE-LETRAS-LICENCIATURA-EM-

LIBRAS.PDF/3E5E55A8-3E8F-4ADA-AD9D-33860C6004A8>. ACESSO EM: 03 DE OUT. DE 2020.

UNIVERSIDADE FEDERAL DO PIAUÍ. PROJETO PEDAGóGICO do CURSO DE LICENCIATURA EM LETRAS-LIBRAS PRESENCIAL. DISPONÍVEL EM:

$<$ HTTPS://WWW.UFPI.BR/ARQUIVOS DOWNLOAD/ARQUIVOS/CDAC/PPCO K_LETRAS_LIBRAS20190712164212.PDF>. ACESSO EM: 03 DE OUT. DE 2020.

UNIVERSIDADE FEDERAL DO RIO GRANDE DO NORTE.

PROJETO PEDAGÓGICO dO CURSO. DISPONÍVEL EM:

<HTTPS://SIGAA.UFRN.BR/SIGAA/PUBLIC/CURSO/PPP.JSF?LC=PT_BR\&ID= 118921874>. ACESSO EM: 03 DE OUT. DE 2020.

UNIVERSIDADE FEDERAL DO RIO DE JANEIRO. CURSO DE GRADUAÇÃo EM LICENCIATURA EM LETRAS - LIBRAS. DISPONÍVEL EM: $<$ HTTPS://SIGA.UFRJ.BR/SIRA/TEMAS/ZIRE/FRAMECONSULTAS.JSP?MAINPA GE=/REPOSITORIO-CURRICULO/33A46672-92A4-F79A-204D193CF502A578.HTML>. ACESSO EM: 03 DE OUT. DE 2020.

UNIVERSIDADE FEDERAL DE RONDÔNIA. PROJETO PolítICO PEDAGÓGICO DO CURSO DE LETRAS - LIBRAS LICENCIATURA. DISPONÍVEL EM: 


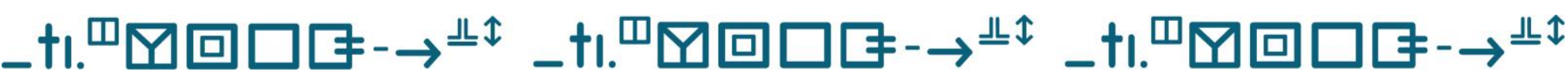

Formação de professores de Libras: o ensino da escrita de sinais nas licenciaturas em Libras

Fabiane Ferreira da Silva Moraes

<HTTP://WWW.DLIBRAS.UNIR.BR/UPLOADS/81818181/PROJETO\%20 PEDAGOGICO\%20DO\%20CURSO\%20DE\%20LETRAS20LIBRAS _REFORMULADO_2017.PDF>. ACESSO EM: 03 DE OUT. DE 2020.

UNIVERSIDADE FEDERAL RURAL DA AMAZÔNIA. PROJETO PEDAGÓGICO DO CURSO DE LETRAS LIBRAS. DISPONIVEEL EM: $<$ HTTPS://LETRASLIBRAS.UFRA.EDU.BR/IMAGES/PPC-LETRASLIBRAS.PDF>. ACESSO EM: 03 DE OUT. DE 2020.

UNIVERSIDADE FEDERAL RURAL DO SEMI-ÁRIDO. CURRÍcULO. DISPONÍVEL EM:

<HTTPS://SIGAA.UFERSA.EDU.BR/SIGAA/PUBLIC/CURSO/CURRICULO.JSF>. ACESSO EM: 03 DE OUT. DE 2020.

UNIVERSIDADE FEDERAL DE SANTA CATARINA. PRojeto POLÍTICO PEDAGÓGICO DO CURSO DE LETRAS LIBRAS. DISPONÍVEL EM: <HTTP://LETRASLIBRAS.PAGINAS.UFSC.BR/FILES/201304/PPPLIBRAS_C URRICULO_2012_FINAL_06-03-2014.PDF>. ACESSO EM: 03 DE OUT. DE 2020.

UNIVERSIDADE FEDERAL DE SANTA CATARINA. CURRÍ́CULO DO CURSO. DISPONÍVEL EM:

$<$ HTTPS://CAGR.SISTEMAS.UFSC.BR/RELATORIOS/CURRICULOCURSO?CURS O=705>. ACESSO EM: 03 DE OUT. DE 2020.

UNIVERSIDADE FEDERAL DO SERGIPE. COMPONENTES CURRICULARES. DISPONIVELL EM: <HTTPS://WWW.SIGAA.UFS.BR/SIGAA/PUBLIC/DEPARTAMENTO/DOCUMEN TOS.JSF?LC=PT BR\&ID=912>.ACESSO EM: 03 DE OUT. DE 2020.

UNIVERSIDADE FEDERAL DO TOCANTINS. MATRIZ CURRICULAR. DISPONÍVEL EM: <HTTP://DOWNLOAD.UFT.EDU.BR/?D=578EF63D1C57-4A99-B5DF- 


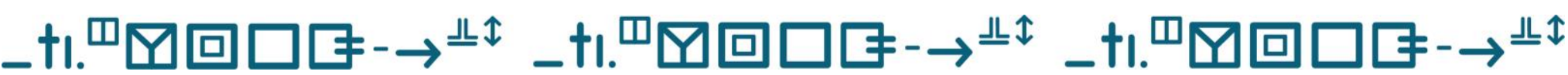

Formação de professores de Libras: o ensino da escrita de sinais nas licenciaturas em Libras

Fabiane Ferreira da Silva Moraes

BEB6D95CB6D0;1.0:MATRIZ\%20CURRICULAR.PDF>. ACESSO EM:

03 DE OUT. DE 2020.

UNIVERSIDADE FEDERAL DE UBERLÂNDIA. FICHA DE

COMPONENTE CURRICULAR. DISPONIVEL EM:

<HTTP://WWW.PORTAL.ILEEL.UFU.BR/SYSTEM/FILES/CONTEUDO/8O_PERI

ODO_-_FACED49696_-_ESCRITA_DE_SINAIS.PDF >. ACESSO EM: 03 DE

OUT. DE 2020.

\section{Publisher}

UNIVERSIDADE FEDERAL dE GOIÁS. CURSOS DE LETRAS: LIBRAS E DE LETRAS: TRADUÇÃO E INTERPRETAÇÃO EM LIBRAS/PORTUGUÊS DA

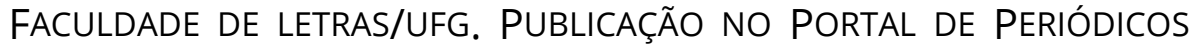
UFG. AS IDEIAS EXPRESSADAS NESTE ARTIGO SÃO DE RESPONSABILIDADE DE SUA AUTORA, NÃO REPRESENTANDO, NECESSARIAMENTE, A OPINIÃO DOS EDITORES OU DA UNIVERSIDADE. 